\title{
Sensibilité des populations d' Aedes Aegypti vis-à-vis des organochlorés, pyréthrinoïdes et des carbamates dans la commune de Natitingou au Nord-Est du Bénin
}

\author{
Yadouleton Anges \\ Klotoe Jean-Robert
}

Ecole Normale Supérieure de Natitingou, Université Nationale des Sciences, Technologies, Ingénierie et Mathématiques (UNSTIM) d'Abomey

Chabi Christophe

Faculé d'Agronomie, Universite de Parakou

Agbanrin Ramziyath

Tchibozo Carine

Agolinou Achaz

Tossou Roland

Centre de Recherches Entomologiques de Cotonou, Cotonou, Benin

\section{Baba-Moussa Lamine}

Laboratoire de Biologie et de Typage Moléculaire en Microbiologie,

Faculté des Sciences et Techniques, Université d'Abomey-Calavi

Doi: 10.19044/esj.2018.v14n33p134 URL:http://dx.doi.org/10.19044/esj.2018.v14n33p134

\begin{abstract}
Insecticide treatments for crop protection have often be cited as the main factor of the resistance selection of Aedes aegypti. This is however the main vector dengue fever. To verify this hypothesis, bioassay tests was performed on adults collected from the field ( 2 urban and 2 rural areas) at Natitingou to assess the susceptibility of dengue vectors to insecticideimpregnated papers (permethrin $0.75 \%$, delthamethrin $0.05 \%$, lambdacyalothrin $0.05 \%$, DDT $4 \%$, and bendiocarb $0.1 \%$ ) following WHO Pesticide Evaluation Scheme. Results from this study showed that Ae. Aegypti populations were resistant to DDT and pyrethroids with $12 \%$ and $38 \%$ respectively as mean of mortality despite the areas of mosquitoes collection. However, they were fully susceptible to bendiocarb. Our study showed that Aedes aegypti populations had developed resistance to organochlorine and pyrethroids, but are susceptible to carbamate. The low mortality rate recorded in all populations of Ae. Aegypti with bendiocarb is encouraging in regards to the use of this insecticide as an alternative in case of outbreak of dengue fever in this district of Benin.
\end{abstract}


Keywords: Aedes aegypti, Insecticides, Resistance, Natitingou, Benin

\section{Résumé}

Les traitements insecticides destinés à la protection des cultures ont été souvent évoqués comme les principaux facteurs de sélection de la résistance chez Aedes aegypti, principal vecteur de la dengue. Pour vérifier cette hypothèse, des collectes larvaires d'Ae. aegypti ont été faites dans deux zones urbaines et rurales de la commune de Natitingou au Nord-Est du Bénin. Les femelles âgées de 2-5 jours issues de ces collectes larvaires ont été mises en contact avec des papiers imprégnés de dichlorodiphényltrichloroéthane (DDT) (4\%), deltaméthrine $(0,05 \%)$, perméthrine $(0,75 \%)$, lambdacyalothrine $(0,5 \%)$, et du bendiocarb $(0,1 \%)$ pour les tests de sensibilité. Il ressort des travaux que les populations d'Ae. Aegypti de la commune de Natitingou ont été resistantes vis-à-vis du DDT et des pyréthrinoïdes, avec un taux moyen de mortalité respectivement de $12 \%$ et de $38 \%$, quelle que soit la zone de provenance du moustique mais elles restent cependant sensibles au bendiocarb. La sensibilité d'Ae. Aegypti vis-á-vis du bendicarb serait un signal indicatif pour utiliser cet insecticide comme alternatif aux pyréthrinoïdes contre Ae. Aegypti en cas d'épidémie de la dengue dans cette commune du Bénin.

Mots-clés: Aedes aegypti, Insecticides, Résistance, Natitingou, Benin

\section{Introduction}

Les maladies à transmission vectorielle, notamment la fièvre jaune et la dengue transmise par Aedes aegypti, constituent de véritables obstacles pour le développement des pays tropicaux (Bhatt et al., 2013 ; Caron et al., 2013). Ce moustique est le vecteur de la fièvre jaune et de la dengue. Il est actif dans plus de 100 pays dans le monde avec une vaste aire de distribution en Afrique, dans les Amériques, en Méditerranée orientale, en Asie du SudEst et dans le Pacifique occidental avec plusieurs cas de décès (Chang et al., 2011; Ellis et al., 2013; Messina et al., 2014 ). Il est hématophage et se trouve dans les habitations humaines du nord au sud du Bénin durant toute l'année, étant présent dans les jarres, boîtes de conserves abandonnées avec des risques de propagation de la fièvre de la dengue (Yadouleton et al., 2018).

Ce moustique transmet une maladie virale causée par un virus de la famille des flaviviridae appartenant à quatre stéréotypes (DEN-1 à - 4). Sa période d'incubation est en moyenne de 4 à 7 jours après la piqûre infectante. De manière générale, on note une manifestation d'un syndrome grippal chez toutes les catégories d'individus (enfants et adultes) touchées par ce virus. Toutefois, la maladie commence généralement de façon abrupte 
avec une forte fièvre accompagnée de maux de tête, de douleurs musculaires et articulaires, de nausées, de vomissements et d'une éruption cutanée (WHO, 2017).

Au cours des cinq dernières années, plusieurs cas de recrudescences de cette fièvre ont été signalés dans plusieurs pays de l'Afrique de l'Ouest, notamment au Burkina en 2016 et 2017 (2680 cas suspects et 20 cas de décès enregistrés), en Côte d'Ivoire en 2017 avec 911 cas suspects notifiés dans les établissements sanitaires d'Abidjan où 311 cas de dengue ont été confirmés comme stéréotype 2 et 3 (WHO, 2017).

Pour réduire l'incidence de la fièvre de la dengue, la lutte antivectorielle basée sur l'utilisation d'insecticides de diverses classes a été une des solutions pour venir à bout de la population d'Ae.aegypti. Cependant, les travaux de Marcombe et al. (2012), Vera-Maloof FZ et al. (2015) ont montré que l'utilisation d'insecticides contre les vecteurs de la dengue a contribué à la sélection de la résistance d'Ae.aegypti. vis-à-vis des pyréthrinoïdes et des organophosphorés.

Au Bénin et dans beaucoup de pays de l'Afrique subsaharienne, Ae. Aegypti partage les mêmes milieux écologiques que 1' Anopheles. Gambiae, le principal vecteur du paludisme. Il se peut que les insecticides utilisés pour le traitement des gîtes larvaires des vecteurs du paludisme en santé publique ou ceux utilisés en agriculture contre les ravageurs des cultures à Natitingou auraient selectionnéla résistance chez les populations d'Ae. Aegypti vis-ávis des organochlorés, pyréthrinoïdes et des carbamates dans la commune de Natitingou au Nord-Est du Bénin.

Ainsi, la présente étude a été initiée afin de connaître le niveau de sensibilité des populations d'Aedes Aegypti vis-à-vis des organochlorés, pyréthrinoïdes et des carbamates dans la commune de Natitingou au Nord-Est du Bénin.

\section{Matériel et méthodes}

La collecte des données a eu lieu dans le département l'Atacora, plus précisément dans la commune de Natitingou $\left(1^{\circ} 23 \mathrm{E}, 10^{\circ} 18 \mathrm{~N}\right)$. Quatre points de collecte ont été choisis dans la commune de Natitingou dont deux en zone urbaine (Ourbouga et Kantaborifa) et deux autres en zone rurale (Tigniti et Yimporima) (Figure 1).

Le choix de la ville de Natitingou se justifie par le caractère agricole de la ville où les paysans, pour le contrôle des ravageurs des cultures, utilisent plusieurs types de pesticides, ceci sans aucun respect des doses recommandées (Yadouleton et al., 2014). 


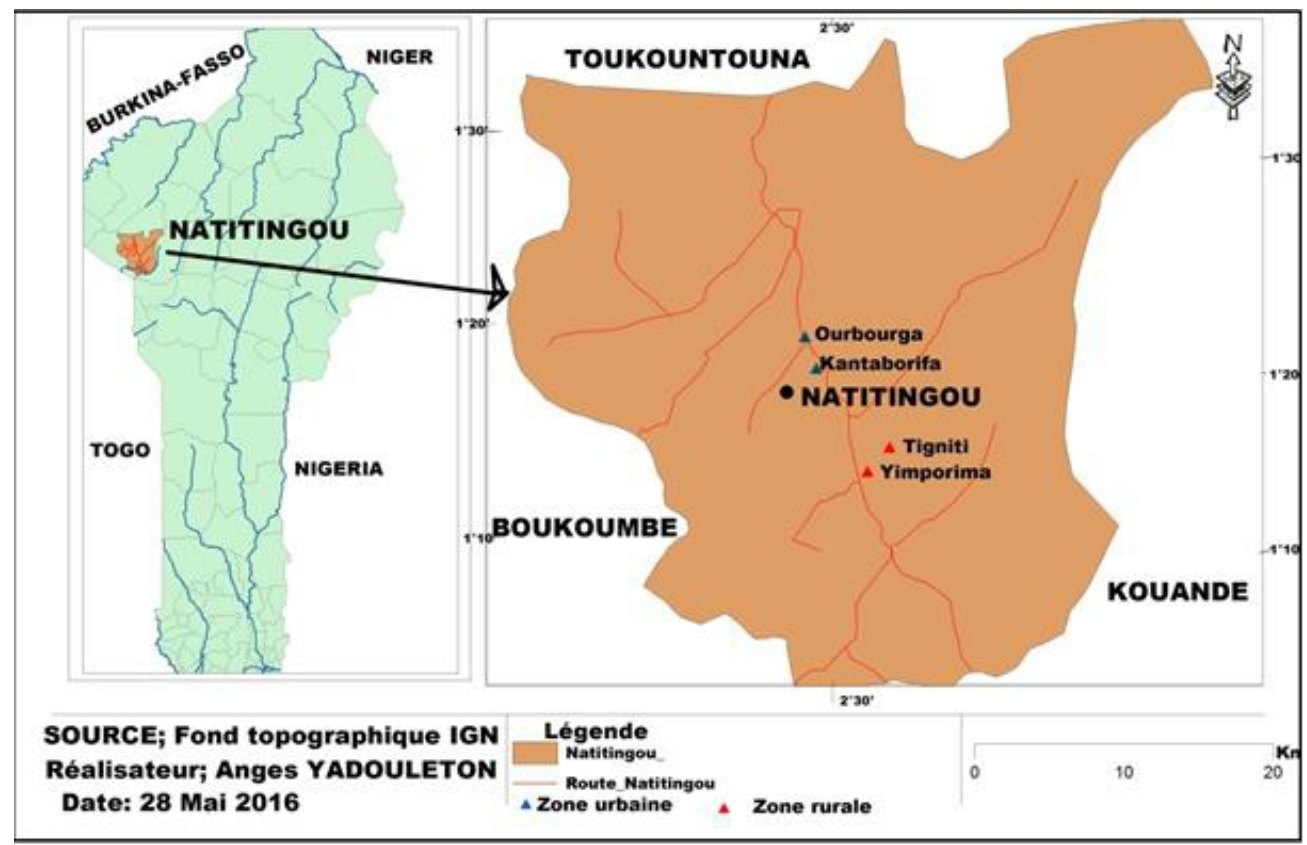

Figure 1. Carte du Bénin indiquant le site d'étude

\section{Echantillonnage des larves}

La récolte des larves a été faite dans les jarres abandonnées, les boîtes de conserve et d' autres récipients usagers. Elles ont été ramenées à l'insectarium du laboratoire d'Entomologie de l'Ecole Normale Supérieure de Natitingou pour élevage. Les adultes femelles âgées de 2 à 5 jours ont été isolées pour être soumises au test de sensibilité.

\section{Tests de sensibilité}

Les tests ont été réalisés avec des papiers imprégnés de permethrine $(0,75 \%)$, deltaméthrine $(0.05 \%)$, lambdacyalothrine $(0.05 \%)$, DDT $(4 \%)$ et du bendiocarb $(0,1 \%)$ en provenance de l'Université Sains Malaysia, un centre collaborateur de l'Organisation Mondiale de la Santé (WHO, 1998). Le DDT a été utilisé pour vérifier la résistance croisée entre les pyréthrinoïdes et les organochlorés d'une part, mais aussi du fait de son utilisation par beaucoup de paysans malgré son interdiction pour lutter contre les ravageurs des cultures dans les zones cotonnières au Bénin (Yadouleton et al., 2011).

Le temps d'exposition des moustiques aux papiers imprégnés a été de 60 minutes et le temps d'observation est de 24 heures. Dès l'exposition des moustiques à l'insecticide, le nombre de moustiques "knocked-down" $(k d)$, c'est-à-dire qui étaient tombés inanimés au fond des tubes OMS, a été noté après 10, 15, 20, 30, 45, 60 minutes. Après les tests, les moustiques morts et vivants ont été mis dans des tubes Eppendorf sur du silicagel et mis à $-20^{\circ} \mathrm{C}$ pour la recherche des mécanismes de résistance. 


\section{Analyse des données}

Les taux de mortalité ont été interprétés selon les critères recommandés par l'OMS (WHO, 1998). Pour une mortalité supérieure ou égale à $97 \%$, on parle d'une population sensible. Lorsque la mortalité est inférieure à $80 \%$, on parle de population résistante mais entre ces deux valeurs, la résistance est suspectée.

Les résultats des tests de sensibilité des populations d'Ae. Aegypti en provenance des quatres points de collecte dans la commune de Ntitingou ont été comparées á la souche de référence au laboratoire SBE, sensible á tous les insecticides.

\section{Résultats}

\section{Sensibilité de Aedes aegypti aux insecticides}

Les femelles d'Ae. Aegypti soumises aux differents papiers impregnés ont montré que la population de ce moustique a été resistante vis-á-vis des organochlorés (Figure 2), et des pyréthrinoïdes (Figure 3-5) avec un taux moyen de mortalité de $12 \%$ et de $38 \%$ respectivement pour les deux familles d'insecticide.

Par contre, ces mêmes populations d'Ae. aegypti issues du site d'étude ont fait preuve d'une grande sensibilité vis-à-vis des carbamates (bendiocarb) avec un taux de mortalité égal à $100 \%$.

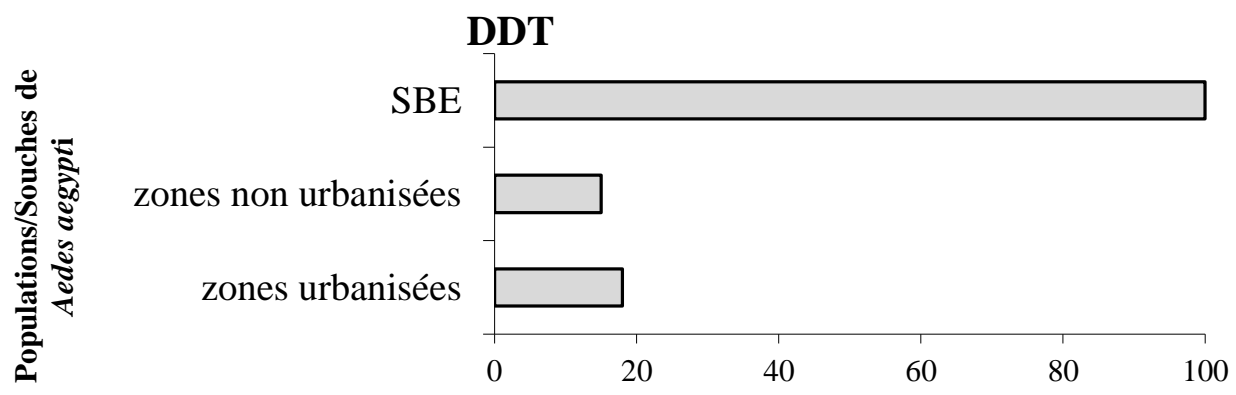

Taux de motalité

Figure 2. Mortalité observée après exposition des populations d' Ae. aegypti au DDT (4\%)

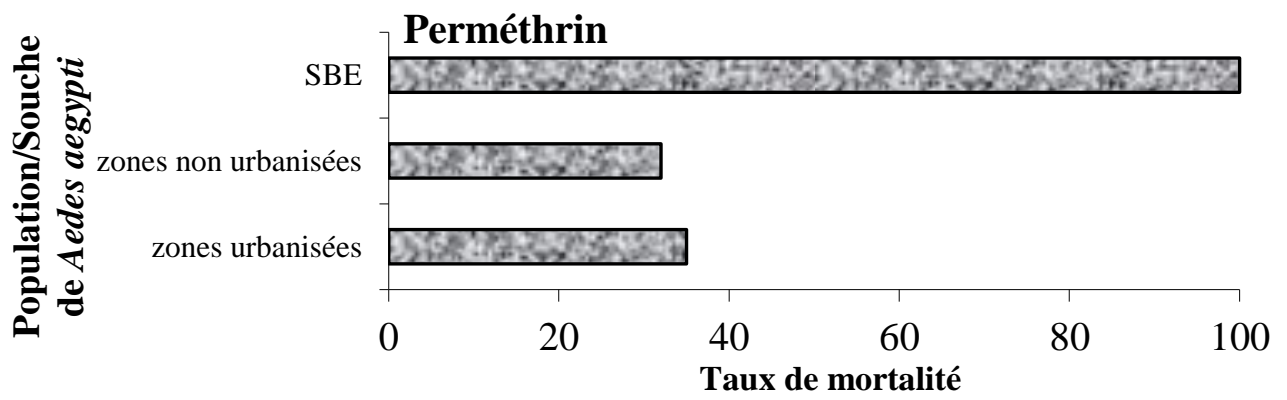

Figure 3. Mortalité observée après exposition des populations d' Ae. Aegypti à la perméthrine 


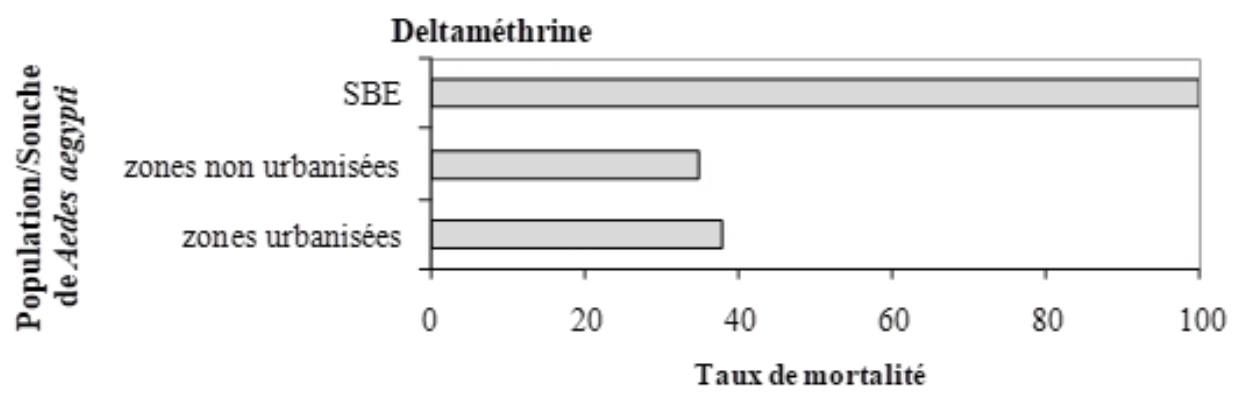

Figure 4. Mortalité observée après exposition des populations de Ae aegypti à la deltaméthrine

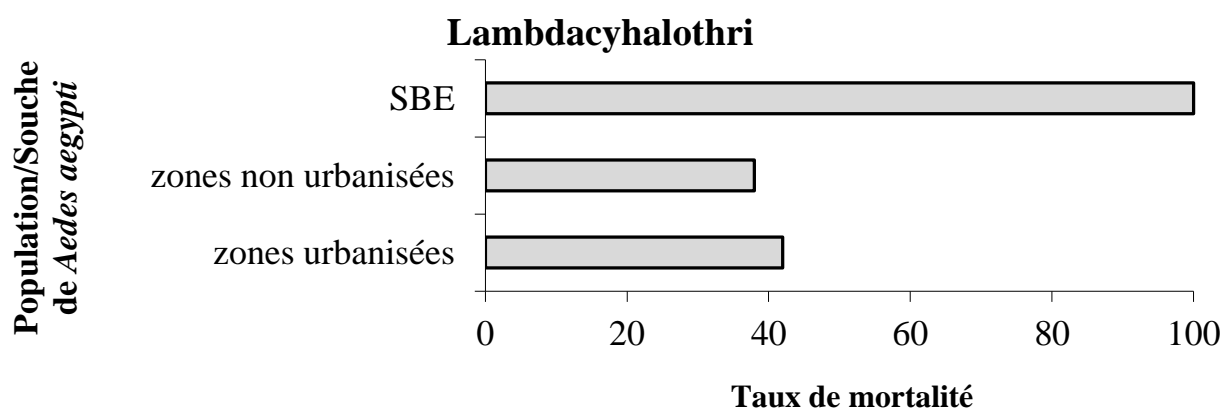

Figure 5. Mortalité observée après exposition des populations d’ Ae. aegypti á la lambdacyhalothrine

\section{Discussion}

Aedes aegypti, principal vecteur de la dengue, a développé une résistance vis-à-vis des organochlorés $(\mathrm{OC})$ et des pyréthrinoïdes $(\mathrm{PY})$ dans la commune de Natitingou au Nord-Est du Bénin. Cette résistance d'Ae. Aegypti aux PY et aux OC serait due á l'utilisation non contrôlée de pesticides chimiques de la part des paysans (Yadouleton et al., 2010). En effet, les pratiques paysannes en matière d'utilisation d'insecticides pour lutter contre les ravageurs des cultures constituent un facteur de sélection d'insectes résistants non seulement au niveau des ravageurs des cultures mais aussi chez les vecteurs du paludisme et des arboviroses comme la dengue (Martin et al., 2010). Après les traitements insecticides, ces derniers entrent en contact avec les gîtes larvaires en exerçant soit une action létale sur les larves de certaines populations d'insectes soit une pression sélective, ce qui conduit progressivement à la sélection de la résistance aux insecticides chez certaines populations de moustiques, notamment chez Ae. Aegypti. (Marcombe et al., 2012). Cela explique la résistance d' Ae.aegypti vis-à-vis des organophosphorés et des pyréthrinoïdes dans la commune de Natitingou au Nord-Est du Bénin. Ces résultats rappelent les résultats des travaux de 
Marcombe et al. (2012), Vera-Maloof FZ et al. (2015) qui ont montré la resistance d'Ae. Aegypti vis-à-vis des PY et des OC.

Même si la résistance au DDT a été observée au sein des populations d'Ae. aegypti, certains auteurs (Aïkpon et al., 2013 ; Etang et al., 2003) pensent que cette résistance pourrait s'expliquer par les pulvérisations massives au DDT faites dans les années 1950s dans le cadre de l'élimination du ver de Guinée et des vecteurs du paludisme en Afrique sub-sahélienne (Joncour, 1959). Le DDT ayant une forte rémanence, il serait évident qu'après plus de 60 années d'utilisation cet insecticide aurait sélectionné la résistance chez les vecteurs de la dengue.

Cependant, la sensiblité des populations d'Ae. Aegypti vis-à-vis du bendiocarb reste un bon signal pour 1 'utilisation de cet insecticide comme alternatif aux pyréthrinoïdes.

Par ailleurs, il serait important de rechercher la mutation knock down resistance au sein des populations Ae. Aegypti vu le taux élevé de mortalité de ce moustique vis-à-vis des pyréthrinoïdes dans cette commune.

\section{Conclusion}

Les résultats des tests de sensibilité ont montré que les populations d' Aedes aegypti ont été résistantes vis-à-vis des pyréthrinoïdes et des organochlorés mais restent sensibles aux carbamates. L'utilisation des carbamates serait donc un alternatif pour pouvoir lutter contre ce moustique en cas d'épidémie de la dengue dans cette commune du Bénin.

\section{References:}

1. Aïkpon, R., Agossa, F., Ossè, R., Oussou, O., Aïzoun, N., Oké-Agbo, F., Akogbeto, M. (2013). Bendiocarb resistance in Anopheles gambiae s.1. populations from Atacora department in Benin, West Africa: a threat for malaria vector control. Parasit Vectors. 6:192.

2. Bhatt, S., Gething, PW., Brady, OJ., Messina, JP., Farlow, AW., Moyes, CL., Drake, JM., Brownstein, JS., Hoen, AG., Sankoh, O., Myers, MF., George, DB., Jaenisch, T., Wint, GR., Simmons, CP., Scott, TW., Farrar, JJ., \& Hay, SI. (2013). The global distribution and burden of dengue. Nature. 496:504-7.

3. Caron, M., Grard, G., Paupy, C., Mombo, IM., Bikie Bi Nso, B., Kassa Kassa, FR. (2013). First evidence of simultaneous circulation of three different dengue virus serotypes in Africa. PLoS ONE.;8:e78030.

4. Chang, MS., Christophel, EM., \& Gopinath, D. (2011). Challenges and future perspective for dengue vector control in the Western Pacific Region. Western Pac Surveill Response. 2:e116. 
5. Ellis, EM., Neatherlin, JC., \& Delorey, M. (2013). A household serosurvey to estimate the magnitude of a dengue outbreak in Mombasa, Kenya. PLoS Negl Trop Dis 2015; 9:e0003733.

6. Etang, J., Manga, L., Chandre, F., Guillet, P., Fondjo, E., Mimpfoundi, R., Toto, JC., \& Fontenille, D. (2003). Insecticide susceptibility status of Anopheles gambiae s.l. (Diptera Culicidae) in the Republic of Cameroon. J Med Entomol. 40: 491-497.

7. Joncour, G. (1959). Lutte anti-palustre au Dahomey. Rapport $n^{\circ} 13$ : Ministère de la Santé Publique.

8. Marcombe, S., Poupardin, R., Darriet, F., Reynaud, S., Bonnet, J., Strode, C. et al. (2009). Exploring the molecular basis of insecticide resistance in the dengue vector Aedes aegypti: a case study in Martinique Island (French West Indies). BMC Genomics. 10:494.

9. Martin, T., Assogba-Komlan, F., Sidick, I., Ahle, V., \& Chandre, F. (2010). An acaricide-treated net to control phytophagous mites. Crop Protection. 5: 470-475.

10. Messina, JP., Brady, OJ., \& Scott, TW. (2014). Global spread of dengue virus types: mapping. the 70 year history. Trends Microbiol. 22:138-46.

11. Vera-Maloof, FZ., Saavedra-Rodriguez, K., Elizondo-Quiroga, AE., Lozano-Fuentes, S., \& Black Iv, WC. (2015). Coevolution of the Ile1,016 and Cys1,534 Mutations in the Voltage Gated Sodium Channel Gene of Aedes aegypti in Mexico. PLOS Neglected Tropical Diseases. 9(12):e0004263.

12. WHO (1998). Evaluation de la santé. Rapport sur la Santé dans le monde. La vie au 21èsiècle, une perspective pour tous, World Health Organisation (ed), Genève Suisse,43-65.

13. WHO (2017). World Malaria Report World Health Organization, Geneva. World Health Organisation (ed), Genève Suisse,108-195.

14. Yadouleton, AW., Padonou, G., Asidi, A., Moiroux, N., Sahabi, B., Corbel, V., Nguessan, R., Gbenou, D., Yacoubou, I., Kinde, G., \& Akogbeto, MC. (2010). Insecticide resistance status in Anopheles gambiae in southern Benin. Malaria Journal. 9: 83-88.

15. Yadouleton, AW., Martin, T., Padonou, G., Chandre, F., Alex, A., Djogbenou, L., Dabiré, R., Aïkpon, R., Glitoh, I., \& Akogbeto, MC. (2011). Cotton pest management strategies on the selection of pyrethroid resistance in Anopheles gambiae populations in northern Benin. Parasites and Vectors. 4:60.

16. Yadouléton, A., Agbanrin, R., Vodounon, C., Padonou, G., Badirou, K., Attolou, R., Ursins, F., Zola, J., Allagbé, H., \& Akogbéto, M. (2014). Seasonal distribution of Aedes aegypti in southern Benin: a risk 
of dengue virus transmission to urban populations. International Journal of Innovation and Applied Studies. 648-654.

17. Yadouleton, A., Tchibozo, C., Agolinou, A., Kpodo, E., Kpodjedo, J., Bankole, H., \& Gbaguidi, F. (2018). Dengue fever immunoglobulin G ( $\operatorname{IgG}$ ) isotype in blood samples from hospital in Benin. World Wide J. Multidisc. Res. Dev. 4(8): 15-18. 